\title{
NORMAL FAMILIES OF ZERO-FREE MEROMORPHIC FUNCTIONS
}

\author{
BINGMAO DENG, MINGLIANG FANG ${ }^{\bowtie}$ and DAN LIU
}

(Received 29 July 2011; accepted 23 September 2011)

Communicated by P. C. Fenton

\begin{abstract}
Let $\mathcal{F}$ be a family of zero-free meromorphic functions in a domain $D$, let $h$ be a holomorphic function in $D$, and let $k$ be a positive integer. If the function $f^{(k)}-h$ has at most $k$ distinct zeros (ignoring multiplicity) in $D$ for each $f \in \mathcal{F}$, then $\mathcal{F}$ is normal in $D$.
\end{abstract}

2010 Mathematics subject classification: primary 30D45.

Keywords and phrases: meromorphic function, normality, zero-free.

\section{Introduction}

Let $D$ be a domain in $\mathbb{C}$ and $\mathcal{F}$ be a family of meromorphic functions in $D$. We say that $\mathcal{F}$ is normal in $D$ (in the sense of Montel) if each sequence $\left\{f_{n}\right\}$ in $\mathcal{F}$ has a subsequence $\left\{f_{n_{j}}\right\}$ that converges locally uniformly on $D$, with respect to the spherical metric, to a meromorphic function or $\infty$ (see Hayman [4], Schiff [9], or Yang [11]). To avoid any confusion, we point out that the spherical metric is applied to the values of the function, not to the points in $D$.

In 1959, Hayman [3] proved the following result.

THEOREM 1. Let $f$ be a nonconstant meromorphic function in $\mathbb{C}$ and $k$ be a positive integer. Then at least one of the functions $f$ and $f^{(k)}-1$ has a zero. Moreover, if $f$ is transcendental, then at least one of the functions $f$ and $f^{(k)}-1$ has infinitely many zeros.

The normality corresponding to Theorem 1 was conjectured by Hayman [5] and confirmed by $\mathrm{Gu}[2]$.

Research supported by the NNSF of China (Grant No. 11071083).

(c) 2011 Australian Mathematical Publishing Association Inc. 1446-7887/2011 \$16.00 
THEOREM 2. Let $k$ be a positive integer and let $\mathcal{F}$ be a family of zero-free meromorphic functions in a domain D. If, for each $f \in \mathcal{F}$, the function $f^{(k)}-1$ has no zeros in $D$, then $\mathcal{F}$ is normal in $D$.

In 1986, Yang [10] extended Theorem 2 as follows.

Theorem 3. Let $\mathcal{F}$ be a family of meromorphic functions defined in a domain $D$ and $h$ be a holomorphic function in $D$ that is not identically zero. If, for each $f \in \mathcal{F}$, the functions $f$ and $f^{(k)}-h$ have no zeros in $D$, then $\mathcal{F}$ is normal in $D$.

Recently, Chang [1] improved Theorem 2 and proved the following result.

THEOREM 4. Let $k$ be a positive integer and $\mathcal{F}$ be a family of zero-free meromorphic functions in a domain $D$ such that, for each $f \in \mathcal{F}$, the function $f^{(k)}-1$ has at most $k$ distinct zeros (ignoring multiplicity) in $D$. Then $\mathcal{F}$ is normal in $D$.

Chang also gave an example to show that the condition that $f^{(k)}-1$ has at most $k$ distinct zeros is best possible.

It is natural to ask whether Theorem 3 remains valid if we replace the hypothesis that $f^{(k)}-h$ has no zeros with the hypothesis that $f^{(k)}-h$ has at most $k$ distinct zeros. In this paper, we use the methods of Chang [1] and of Pang et al. [7] to give an affirmative answer to the question. Here is our main result.

THeOREM 5. Let $\mathcal{F}$ be a family of zero-free meromorphic functions in a domain D, let $h$ be a holomorphic function in $D$ that is not identically zero, and let $k$ be a positive integer. If the function $f^{(k)}-h$ has at most $k$ distinct zeros (ignoring multiplicity) in $D$ for each $f \in \mathcal{F}$, then $\mathcal{F}$ is normal in $D$.

Example 6. Suppose that $\mathcal{F}=\left\{f_{n}(z)=1 /(n z): n=1,2,3, \ldots\right\}$, that $D=\{z:|z|<1\}$, and that $h(z)=1 / z^{k+1}$, where $k$ is a positive integer. Then, for any $f_{n} \in \mathcal{F}$, the function $f_{n}^{(k)}-h$ has only one zero in $D$, but $\mathcal{F}$ is not normal in $D$. This shows that Theorem 5 is not valid if the function $h$ is allowed to be meromorphic.

Example 7. Suppose that $\mathcal{F}=\left\{f_{n}(z)=1 /(n z): n \geq k ! 2^{(k+1)}+1\right\}$, that $D=\{z:|z|<1\}$, and that $h(z)=1 /(z-1)^{k+1}$, where $k$ is a positive integer. Then, for any $f_{n} \in \mathcal{F}$, the function $f_{n}^{(k)}-h$ has $k+1$ distinct zeros in $D$, but $\mathcal{F}$ is not normal in $D$. This shows that the condition in Theorem 5 that $f^{(k)}-h$ has at most $k$ distinct zeros (ignoring multiplicity) in $D$ is best possible.

\section{Some lemmas}

For the proof of Theorem 5, we require the following results.

Lemma 8 [8, 12]. Let $\alpha \in \mathbb{R}$ satisfy $-1<\alpha<\infty$ and let $\mathcal{F}$ be a family of zero-free meromorphic functions in a domain $D$. If $\mathcal{F}$ is not normal at $z_{0} \in D$, then there exist points $z_{j} \in D$ tending to $z_{0}$, functions $f_{j} \in \mathcal{F}$, positive numbers $\rho_{j}$ tending to 0 , and a nonconstant zero-free meromorphic function $g$ of order at most two such that

$$
g_{n}(\xi)=\rho_{j}^{-\alpha} f_{j}\left(z_{j}+\rho_{j} \xi\right) \rightarrow g(\xi)
$$

locally uniformly in $\xi$ in $\mathbb{C}$, with respect to the spherical metric. 
Lemma 9 [7]. Let $f$ be a transcendental meromorphic function of finite order, a be a polynomial that is not identically zero, and $k$ be a positive integer. If the function $f$ has no zeros, then the function $f^{(k)}-a$ has infinitely many zeros.

Lemma 10 [1]. Let $f$ be a nonconstant zero-free rational function and $k$ be $a$ positive integer. Then the function $f^{(k)}-1$ has at least $k+1$ distinct zeros (ignoring multiplicity) in $\mathbb{C}$.

Using the method of Chang [1], we obtain the following lemma.

LEMMA 11. Let $f$ be a nonconstant zero-free rational function, a be a polynomial that is not identically zero, and $k$ be a positive integer. Then the function $f^{(k)}-a$ has at least $k+1$ distinct zeros (ignoring multiplicity) in $\mathbb{C}$.

Proof. If $\operatorname{deg} a=0$, then $a$ is constant, and the result follows from Lemma 10.

Now we suppose that $\operatorname{deg} a>0$. Since $f$ is a nonconstant zero-free rational function, $f$ is not a polynomial, and hence has at least one finite pole. Further, by calculation, the function $f^{(k)}-a$ has at least one zero in $\mathbb{C}$. Thus, we can write

$$
\begin{gathered}
a(z)=A \prod_{i=1}^{m}\left(z+v_{i}\right)^{m_{i}}, \\
f(z)=\frac{C_{1}}{\prod_{i=1}^{n}\left(z+z_{i}\right)^{n_{i}}}, \\
f^{(k)}(z)=a(z)+\frac{C_{2} \prod_{i=1}^{s}\left(z+w_{i}\right)^{l_{i}}}{\prod_{i=1}^{n}\left(z+z_{i}\right)^{n_{i}+k}},
\end{gathered}
$$

where $A, C_{1}$, and $C_{2}$ are nonzero constants, $m, n, s, l_{i}, m_{i}$, and $n_{i}$ are positive integers, the $v_{i}$ (when $1 \leq i \leq m$ ) are distinct complex numbers, and the $w_{i}$ (when $1 \leq i \leq s$ ) and $z_{i}$ (when $1 \leq i \leq n$ ) are distinct complex numbers.

Set $M=\sum_{i=1}^{m} m_{i}$ and $N=\sum_{i=1}^{n} n_{i}$. Then $\operatorname{deg} a=M \geq 1$. By induction, we deduce from (2) that

$$
f^{(k)}(z)=\frac{P_{k}(z)}{\prod_{i=1}^{n}\left(z+z_{i}\right)^{n_{i}+k}},
$$

where $P_{k}$ is a polynomial of degree $(n-1) k$. Thus, by (1), (3), and (4),

$$
A \prod_{i=1}^{m}\left(z+v_{i}\right)^{m_{i}} \prod_{i=1}^{n}\left(z+z_{i}\right)^{n_{i}+k}+C_{2} \prod_{i=1}^{s}\left(z+w_{i}\right)^{l_{i}}=P_{k}(z) .
$$

It follows that

$$
\sum_{i=1}^{s} l_{i}=\sum_{i=1}^{n}\left(n_{i}+k\right)+\sum_{i=1}^{m} m_{i}=n k+N+M
$$

and $C_{2}=-A$. Thus, by (5),

$$
\prod_{i=1}^{m}\left(1+v_{i} t\right)^{m_{i}} \prod_{i=1}^{n}\left(1+z_{i} t\right)^{n_{i}+k}-\prod_{i=1}^{s}\left(1+w_{i} t\right)^{l_{i}}=t^{k+N+M} Q(t),
$$


where $Q(t)=t^{(n-1) k} P_{k}(1 / t) / A$. Then $Q$ is a polynomial of degree less than $(n-1) k$, and it follows that

$$
\frac{\prod_{i=1}^{m}\left(1+v_{i} t\right)^{m_{i}} \prod_{i=1}^{n}\left(1+z_{i} t\right)^{n_{i}+k}}{\prod_{i=1}^{s}\left(1+w_{i} t\right)^{l_{i}}}=1+\frac{t^{k+N+M} Q(t)}{\prod_{i=1}^{s}\left(1+w_{i} t\right)^{l_{i}}} .
$$

Note that, for $t$ near 0 ,

$$
\frac{t^{k+N+M} Q(t)}{\prod_{i=1}^{s}\left(1+w_{i} t\right)^{l_{i}}}=t^{k+N+M}\left(a_{0}+a_{1} t+\cdots\right),
$$

where $a_{0} \neq 0$. Logarithmic differentiation of both sides of (6) and (7) shows that

$$
\sum_{i=1}^{m} \frac{m_{i} v_{i}}{1+v_{i} t}+\sum_{i=1}^{n} \frac{\left(n_{i}+k\right) z_{i}}{1+z_{i} t}-\sum_{i=1}^{s} \frac{l_{i} w_{i}}{1+w_{i} t}=O\left(t^{k+N+M-1}\right) \quad \text { as } t \rightarrow 0 .
$$

Set

$$
S_{1}=\left\{v_{1}, v_{2}, \ldots, v_{m}\right\} \cap\left\{z_{1}, z_{2}, \ldots, z_{n}\right\}
$$

and

$$
S_{2}=\left\{v_{1}, v_{2}, \ldots, v_{m}\right\} \cap\left\{w_{1}, w_{2}, \ldots, w_{s}\right\}
$$

We consider four cases.

Case 1: $S_{1}=S_{2}=\emptyset$. Let $z_{n+i}=v_{i}$ when $1 \leq i \leq m$ and

$$
N_{i}= \begin{cases}n_{i}+k & \text { when } 1 \leq i \leq n \\ m_{i-n} & \text { when } n+1 \leq i \leq n+m\end{cases}
$$

In this case, (8) may be rewritten:

$$
\sum_{i=1}^{n+m} \frac{N_{i} z_{i}}{1+z_{i} t}-\sum_{i=1}^{s} \frac{l_{i} w_{i}}{1+w_{i} t}=O\left(t^{k+N+M-1}\right) \quad \text { as } t \rightarrow 0 .
$$

Comparing the coefficients of $t^{j}$ when $j=0,1, \ldots, k+N+M-2$ in (9), we deduce that

$$
\sum_{i=1}^{n+m} N_{i} z_{i}^{j}-\sum_{i=1}^{s} l_{i} w_{i}^{j}=0 \quad \forall j \in\{1,2, \ldots, k+N+M-1\} .
$$

Let $z_{n+m+i}=w_{i}$ when $1 \leq i \leq s$. Noting that $\sum_{i=1}^{n+m} N_{i}-\sum_{i=1}^{s} l_{i}=0$ and using (10), we deduce that the system of linear equations

$$
\sum_{i=1}^{n+m+s} z_{i}^{j} x_{i}=0
$$

where $0 \leq j \leq k+N+M-1$, has a nonzero solution

$$
\left(x_{1}, \ldots, x_{n+m}, x_{n+m+1}, \ldots, x_{n+m+s}\right)=\left(N_{1}, \ldots, N_{n+m},-l_{1}, \ldots,-l_{s}\right) .
$$


If $k+N+M \geq n+m+s$, then the determinant $\operatorname{det}\left(z_{i}^{j}\right)_{(n+m+s) \times(n+m+s)}$ of the coefficients of the system of equations (11), where $0 \leq j \leq n+m+s-1$, is equal to zero, by Cramer's rule (see for instance [6]). However, the $z_{i}$ are distinct complex numbers when $1 \leq i \leq n+m+s$, and the determinant is a Vandermonde determinant, so cannot be 0 (see [6]), which is a contradiction.

Hence, we conclude that $k+N+M<n+m+s$. It follows from this and the two inequalities $N=\sum_{i=1}^{n} n_{i} \geq n$ and $M=\sum_{i=1}^{m} m_{i} \geq m$ that $s \geq k+1$.

Case 2: $S_{1} \neq \emptyset$ and $S_{2}=\emptyset$. Without loss of generality, we may and shall assume that $S_{1}=\left\{v_{1}, v_{2}, \ldots, v_{M_{1}}\right\}$. Thus, $v_{i}=z_{i}$ when $1 \leq i \leq M_{1}$. Let $M_{3}=m-M_{1}$. We consider two subcases.

Subcase 2.1: $M_{3} \geq 1$. Set $z_{n+i}=v_{M_{1}+i}$ when $1 \leq i \leq M_{3}$. If $M_{1}<n$, then set

$$
N_{i}= \begin{cases}n_{i}+m_{i}+k & \text { when } 1 \leq i \leq M_{1}, \\ n_{i}+k & \text { when } M_{1}+1 \leq i \leq n, \\ m_{M_{1}-n+i} & \text { when } n+1 \leq i \leq n+M_{3} .\end{cases}
$$

If $M_{1}=n$, then set

$$
N_{i}= \begin{cases}n_{i}+m_{i}+k & \text { when } 1 \leq i \leq M_{1}=n, \\ m_{M_{1}-n+i} & \text { when } n+1 \leq i \leq n+M_{3} .\end{cases}
$$

Subcase 2.2: $M_{3}=0$. If $M_{1}<n$, then set

$$
N_{i}= \begin{cases}n_{i}+m_{i}+k & \text { when } 1 \leq i \leq M_{1}, \\ n_{i}+k & \text { when } M_{1}+1 \leq i \leq n .\end{cases}
$$

If $M_{1}=n$, then set

$$
N_{i}=n_{i}+m_{i}+k \quad \text { when } \leq i \leq M_{1}=n .
$$

In both subcases, (8) may be rewritten:

$$
\sum_{i=1}^{n+M_{3}} \frac{N_{i} z_{i}}{1+z_{i} t}-\sum_{i=1}^{s} \frac{l_{i} w_{i}}{1+w_{i} t}=O\left(t^{k+N+M-1}\right) \quad \text { as } t \rightarrow 0,
$$

where $0 \leq M_{3} \leq m-1$. Using the argument of Case 1, we deduce that $s \geq k+1$.

Case 3: $S_{1}=\emptyset$ and $S_{2} \neq \emptyset$. Without loss of generality, we may and shall assume that $S_{2}=\left\{v_{1}, v_{2}, \ldots, v_{M_{2}}\right\}$. Thus, $v_{i}=w_{i}$ when $1 \leq i \leq M_{2}$. Let $M_{4}=m-M_{2}$. We consider two subcases.

Case 3.1: $M_{4} \geq 1$. Set $w_{s+i}=v_{M_{2}+i}, 1 \leq i \leq M_{4}$. If $M_{2}<s$, then set

$$
L_{i}= \begin{cases}l_{i}-m_{i} & \text { when } 1 \leq i \leq M_{2}, \\ l_{i} & \text { when } M_{2}+1 \leq i \leq s, \\ -m_{M_{2}-s+i} & \text { when } s+1 \leq i \leq s+M_{4} .\end{cases}
$$


If $M_{2}=s$, set

$$
L_{i}= \begin{cases}l_{i}-m_{i} & \text { when } 1 \leq i \leq M_{2}=s, \\ -m_{M_{2}-s+i} & \text { when } s+1 \leq i \leq s+M_{4} .\end{cases}
$$

Case 3.2: $M_{4}=0$. If $M_{2}<s$, then set

$$
L_{i}= \begin{cases}l_{i}-m_{i} & \text { when } 1 \leq i \leq M_{2}, \\ l_{i} & \text { when } M_{2}+1 \leq i \leq s .\end{cases}
$$

If $M_{2}=s$, then set

$$
L_{i}=l_{i}-m_{i} \quad \text { when } 1 \leq i \leq M_{2}=s .
$$

In both subcases, (8) may be rewritten:

$$
\sum_{i=1}^{n} \frac{n_{i} z_{i}}{1+z_{i} t}-\sum_{i=1}^{s+M_{4}} \frac{L_{i} w_{i}}{1+w_{i} t}=O\left(t^{k+N+M-1}\right) \quad \text { as } t \rightarrow 0,
$$

where $0 \leq M_{4} \leq m-1$. Using the argument of Case 1 , we deduce that $s \geq k+1$.

Case 4: $S_{1} \neq \emptyset$ and $S_{2} \neq \emptyset$. Without loss of generality, we may and shall assume that $S_{1}=\left\{v_{1}, v_{2}, \ldots, v_{M_{1}}\right\}, S_{2}=\left\{w_{1}, w_{2}, \ldots, w_{M_{2}}\right\}$, and $v_{i}=z_{i}$ when $1 \leq i \leq M_{1}$ and $w_{i}=v_{M_{1}+i}$ when $1 \leq i \leq M_{2}$. Set $M_{5}=m-M_{2}-M_{1}$. We consider two subcases.

Case 4.1: $M_{5} \geq 1$. Set $z_{n+i}=v_{M_{1}+M_{2}+i}, 1 \leq i \leq M_{5}$. If $M_{1}<n$, then set

$$
N_{i}= \begin{cases}n_{i}+m_{i}+k & \text { when } 1 \leq i \leq M_{1} \\ n_{i}+k & \text { when } M_{1}+1 \leq i \leq n \\ m_{M_{1}+M_{2}-n+i} & \text { when } n+1 \leq i \leq n+M_{5}\end{cases}
$$

If $M_{1}=n$, then set

$$
N_{i}= \begin{cases}n_{i}+m_{i}+k & \text { when } 1 \leq i \leq M_{1}=n \\ m_{M_{1}+M_{2}-n+i} & \text { when } n+1 \leq i \leq n+M_{5}\end{cases}
$$

If $M_{2}<s$, then set

$$
L_{i}= \begin{cases}l_{i}-m_{M_{1}+i} & \text { when } 1 \leq i \leq M_{2} \\ l_{i} & \text { when } M_{2}+1 \leq i \leq s .\end{cases}
$$

If $M_{2}=s$, then set

$$
L_{i}=l_{i}-m_{M_{1}+i} \quad \text { when } 1 \leq i \leq M_{2}=s .
$$

Case 4.2: $M_{5}=0$. If $M_{1}<n$, then set

$$
N_{i}= \begin{cases}n_{i}+m_{i}+k & \text { when } 1 \leq i \leq M_{1}, \\ n_{i}+k & \text { when } M_{1}+1 \leq i \leq n .\end{cases}
$$


If $M_{1}=n$, then set

$$
N_{i}=n_{i}+m_{i}+k \quad \text { when } 1 \leq i \leq M_{1}=n .
$$

And, if $M_{2}<s$, set

$$
L_{i}= \begin{cases}l_{i}-m_{M_{1}+i} & \text { when } 1 \leq i \leq M_{2}, \\ l_{i} & \text { when } M_{2}+1 \leq i \leq s .\end{cases}
$$

If $M_{2}=s$, then set

$$
L_{i}=l_{i}-m_{M_{1}+i} \quad \text { when } 1 \leq i \leq M_{2}=s .
$$

In both subcases, (8) may be rewritten:

$$
\sum_{i=1}^{n+M_{5}} \frac{N_{i} z_{i}}{1+z_{i} t}-\sum_{i=1}^{s} \frac{L_{i} w_{i}}{1+w_{i} t}=O\left(t^{k+N+M-1}\right) \quad \text { as } t \rightarrow 0,
$$

where $0 \leq M_{5} \leq m-2$. Using the argument of Case 1 , we deduce that $s \geq k+1$.

This completes the proof of Lemma 11.

\section{Proof of Theorem 5}

First we show that $\mathcal{F}$ is normal on the set $D^{\prime}$, defined to be $\{z \in D: h(z) \neq 0\}$. Suppose that $\mathcal{F}$ is not normal at $z_{0} \in D^{\prime}$. We may assume that $D$ is the $\operatorname{disc} \Delta(0,1)$ with center 0 and radius 1 , and that $h\left(z_{0}\right)=1$. By Lemma 8 , there exist points $z_{j} \in D$ tending to $z_{0}$, functions $f_{j} \in \mathcal{F}$, positive numbers $\rho_{j}$ tending to 0 , and a nonconstant zero-free meromorphic function $g$ of order at most two such that

$$
g_{n}(\xi)=\frac{f_{n}\left(z_{n}+\rho_{n} \xi\right)}{\rho_{n}^{k}} \rightarrow g(\xi),
$$

locally uniformly in $\xi$ in $\mathbb{C}$ with respect to the spherical metric.

We claim that the function $g^{(k)}-1$ has at most $k$ distinct zeros. With a view to a contradiction, suppose that $g^{(k)}-1$ has $k+1$ distinct zeros $\xi_{j}$ when $1 \leq j \leq k+1$. By Lemma $8, g^{(k)}$ is not identically 1 . By Hurwitz's theorem and because

$$
g_{n}^{(k)}(\xi)-h\left(z_{n}+\rho_{n} \xi\right)=f_{n}^{(k)}\left(z_{n}+\rho_{n} \xi\right)-h\left(z_{n}+\rho_{n} \xi\right) \rightarrow g^{(k)}(\xi)-1
$$

as $n \rightarrow \infty$, there exist points $\xi_{n, j}$ when $j=1,2, \ldots, k+1$ such that $\xi_{n, j} \rightarrow \xi_{j}$ and

$$
f_{n}^{(k)}\left(z_{n}+\rho_{n} \xi_{n, j}\right)=h\left(z_{n}+\rho_{n} \xi_{n, j}\right) .
$$

However, $f_{n}^{(k)}(z)=h(z)$ has at most $k$ distinct roots in $D$, and $z_{n}+\rho_{n} \xi_{n, j} \rightarrow z_{0}$, which is a contradiction, and proves our claim.

By Lemma 9, $g$ is a rational function. But this contradicts Lemma 10, which shows that $\mathcal{F}$ is normal in $D^{\prime}$. 
We now prove that $\mathcal{F}$ is normal at points $z$ where $h(z)=0$. By making standard normalizations, we may assume that

$$
h(z)=z^{m}+a_{m+1} z^{m+1}+\cdots=z^{m} b(z) \quad \forall z \in \Delta,
$$

where $m \geq 1, b(0)=1$, and $h(z) \neq 0$ when $0<|z|<1$. Let

$$
\mathcal{F}_{1}:=\left\{F: F(z)=\frac{f(z)}{z^{m}}, f \in \mathcal{F}\right\} .
$$

For all $f \in \mathcal{F}$, the function $f$ has no zeros; hence, for all $F \in \mathcal{F}_{1}$, the function $F$ has no zeros, and 0 is a pole of $F$ with multiplicity at least $m$. We shall prove that $\mathcal{F}_{1}$ is normal at 0 . Suppose otherwise: then, by Lemma 8, there exist points $z_{j} \in \Delta$ tending to 0 , functions $F_{j} \in \mathcal{F}$, positive numbers $\rho_{j}$ tending to 0 , and a nonconstant zero-free meromorphic function $g$ of order at most two such that

$$
g_{n}(\xi)=\frac{F_{n}\left(z_{n}+\rho_{n} \xi\right)}{\rho_{n}^{k}} \rightarrow g(\xi),
$$

locally uniformly on $\mathbb{C}$ with respect to the spherical metric. We distinguish two cases, following Pang et al. [7].

Case 1: $\left(z_{n} / \rho_{n}\right)$ has a convergent subsequence. We still denote the subsequence by $\left(z_{n} / \rho_{n}\right)$ and its limit by $\alpha$. Let $\tilde{g}(\xi)=g(\xi-\alpha)$. Then

$$
\frac{F_{n}\left(\rho_{n} \xi\right)}{\rho_{n}^{k}}=\frac{F_{n}\left(z_{n}+\rho_{n}\left(\xi-z_{n} / \rho_{n}\right)\right)}{\rho_{n}^{k}} \rightarrow g(\xi-\alpha)=\tilde{g}(\xi),
$$

the convergence being locally uniform in $\xi$ in $\mathbb{C}$ with respect to the spherical metric, hence uniform on compact subsets of $\mathbb{C}$ disjoint from the poles of $\tilde{g}$. Clearly, $\tilde{g}$ has no zeros, and the pole of $\tilde{g}$ at 0 has order at least $m$. Now define $G_{n}(\xi)=f_{n}\left(\rho_{n} \xi\right) / \rho_{n}^{k+m}$ and $G(\xi)=\xi^{m} \tilde{g}(\xi)$. Then

$$
G_{n}(\xi)=\frac{\left(\rho_{n} \xi\right)^{m} F_{n}\left(\rho_{n} \xi\right)}{\rho_{n}^{k+m}} \rightarrow \xi^{m} \tilde{g}(\xi)=G(\xi),
$$

uniformly on compact subsets of $\mathbb{C}$ disjoint from the poles of $\tilde{g}$. Since $\tilde{g}$ has a pole of order at least $m$ at 0 , it follows that $G(0) \neq 0$; since $\tilde{g}$ has no zeros, it follows that $G$ has no zeros. Further,

$$
\lim _{n \rightarrow \infty} \frac{h\left(\rho_{n} \xi\right)}{\rho_{n}^{m}}=\xi^{m}
$$

uniformly on compact subsets of $\mathbb{C}$. So,

$$
G_{n}^{(k)}(\xi)-\frac{h\left(\rho_{n} \xi\right)}{\rho_{n}^{k}}=\frac{f_{n}^{(k)}\left(\rho_{n} \xi\right)-h\left(\rho_{n} \xi\right)}{\rho_{n}^{m}} \rightarrow G^{(k)}(\xi)-\xi^{m} .
$$

Since $f_{n}^{(k)}-h$ has at most $k$ distinct zeros in the ball $\Delta\left(z_{0}, \delta\right)$ with center $z_{0}$ and radius $\delta$, as discussed above, the equation $G^{(k)}(\xi)=\xi^{m}$ has at most $k$ distinct roots in $\mathbb{C}$.

However, by Lemma 9, $G$ is a rational function, which contradicts Lemma 11. 
Case 2. $\left(z_{n} / \rho_{n}\right)$ has a subsequence tending to $\infty$. We still denote the subsequence by $\left(z_{n} / \rho_{n}\right)$. By simple calculation,

$$
\begin{aligned}
F^{(k)}(z) & =\frac{f^{(k)}(z)}{z^{m}}-\sum_{l=1}^{k} C_{k}^{l} \frac{\left(z^{m}\right)^{(l)} f^{(k-l)}(z)}{z^{m}} \\
& =\frac{f^{(k)}(z)}{z^{m}}-\sum_{l=1}^{k} C_{l} f^{(k-l)}(z) \frac{1}{z^{l}},
\end{aligned}
$$

where

$$
C_{l}= \begin{cases}C_{k}^{l} m(m-1) \cdots(m-l+1) & \text { when } l \leq m, \\ 0 & \text { when } l>m .\end{cases}
$$

From (12) and the identity $\rho_{n}^{l} g_{n}^{(k-l)}(\xi)=F_{n}^{(k-l)}\left(z_{n}+\rho_{n} \xi\right)$, we obtain

$$
\begin{aligned}
g_{n}^{(k)}(\xi) & =F_{n}^{(k)}\left(z_{n}+\rho_{n} \xi\right) \\
& =\frac{f_{n}^{(k)}\left(z_{n}+\rho_{n} \xi\right)}{\left(z_{n}+\rho_{n} \xi\right)^{m}}-\sum_{l=1}^{k} C_{l} F_{n}^{(k-l)}\left(z_{n}+\rho_{n} \xi\right) \frac{1}{\left(z_{n}+\rho_{n} \xi\right)^{l}} \\
& =\frac{f_{n}^{(k)}\left(z_{n}+\rho_{n} \xi\right)}{\left(z_{n}+\rho_{n} \xi\right)^{m}}-\sum_{l=1}^{k} C_{l} g_{n}^{(k-l)}(\xi) \frac{1}{\left(z_{n} / \rho_{n}+\xi\right)^{l}}
\end{aligned}
$$

Hence,

$$
\frac{f_{n}^{(k)}\left(z_{n}+\rho_{n} \xi\right)}{h\left(z_{n}+\rho_{n} \xi\right)}=\left[g_{n}^{(k)}(\xi)+\sum_{l=1}^{k} g_{n}^{(k-l)}(\xi) \frac{C_{l}}{\left(z_{n} / \rho_{n}+\xi\right)^{l}}\right] \frac{1}{b\left(z_{n}+\rho_{n} \xi\right)}
$$

Now $\lim _{n \rightarrow \infty} b\left(z_{n}+\rho_{n} \xi\right)=1$ and $\lim _{n \rightarrow \infty} 1 /\left(z_{n} / \rho_{n}+\xi\right)=0$. So,

$$
\frac{f_{n}^{(k)}\left(z_{n}+\rho_{n} \xi\right)-h\left(z_{n}+\rho_{n} \xi\right)}{h\left(z_{n}+\rho_{n} \xi\right)} \rightarrow g^{(k)}(\xi)-1,
$$

uniformly on compact subsets of $\mathbb{C}$ disjoint from the poles of $g$.

Since $F$ has no zeros and $f^{(k)}-h$ has at most $k$ distinct zeros, as discussed in Case 1 , we see that $g$ has no zeros and $g^{(k)}-1$ has at most $k$ distinct zeros. However, by Lemma $9, g$ is a rational function, and this contradicts Lemma 10.

We have thus proved that $\mathcal{F}_{1}$ is normal at 0 . It remains to prove that $\mathcal{F}$ is normal at 0 . Since $\mathcal{F}_{1}$ is normal at 0 and $F(0)=\infty$ for each $F \in \mathcal{F}_{1}$, there exists $\delta>0$ such that $|F(z)| \geq 1$ for all $F \in \mathcal{F}_{1}$ and all $z \in \Delta(0, \delta)$. If $f \in \mathcal{F}$, then $f$ has no zeros in $\Delta(0, \delta)$, so $1 / f$ is analytic in $\Delta(0, \delta)$. Therefore,

$$
\left|\frac{1}{f(z)}\right|=\left|\frac{1}{F(z)} \frac{1}{z^{m}}\right| \leq \frac{2^{m}}{\delta^{m}} \quad \forall z \in \Delta\left(0, \frac{1}{2} \delta\right)
$$

for all $f \in \mathcal{F}$. By the maximum principle and Montel's theorem, $\mathcal{F}$ is normal at 0 , and thus $\mathcal{F}$ is normal in $D$. This completes the proof of Theorem 5. 
REMARK 12. In the proof of Theorem 5, we just use a very special case of Lemma 11, namely, when $a(z)=z^{m}$.

\section{Acknowledgement}

The authors thank the referee for several helpful suggestions.

\section{References}

[1] J. M. Chang, 'Normality and quasinormality of zero-free meromorphic functions', Acta Math. Sin. (Engl. Ser.), to appear.

[2] Y. X. Gu, 'A criterion for normality of families of meromorphic functions', Sci. Sin. 1 (1979), 267-274.

[3] W. K. Hayman, 'Picard values of meromorphic functions and their derivatives', Ann. of Math. (2) 70 (1959), 9-42.

[4] W. K. Hayman, Meromorphic Functions (Clarendon Press, Oxford, 1964).

[5] W. K. Hayman, Research Problems in Function Theory (Athlone Press, London, 1967).

[6] L. Mirsky, An Introduction to Linear Algebra (Clarendon Press, Oxford, 1955).

[7] X. C. Pang, D. G. Yang and L. Zalcman, 'Normal families of meromorphic functions whose derivatives omit a function', Comput. Methods Funct. Theory 2 (2002), 257-265.

[8] X. C. Pang and L. Zalcman, 'Normal families and shared values', Bull. Lond. Math. Soc. 32 (2000), 325-331.

[9] J. Schiff, Normal Families (Springer, Berlin-Heidelberg-New York, 1993).

[10] L. Yang, 'Normality for families of meromorphic functions', Sci. Sin. 29 (1986), 1263-1274.

[11] L. Yang, Value Distribution Theory (Springer, Berlin-Heidelberg-New York, 1993).

[12] L. Zalcman, 'Normal families: new perspectives', Bull. Amer. Math. Soc. 35 (1998), 215-230.

BINGMAO DENG, Institute of Applied Mathematics, South China Agricultural University, Guangzhou 510642, PR China e-mail: dbmao2012@163.com

MINGLIANG FANG, Institute of Applied Mathematics, South China Agricultural University, Guangzhou 510642, PR China e-mail: mlfang@scau.edu.cn

DAN LIU, Institute of Applied Mathematics, South China Agricultural University, Guangzhou 510642, PR China e-mail: liudan@scau.edu.cn 